\title{
Presença de Loxosceles similis Moenkhaus, 1898 (Araneae, Sicariidae) na Serra da Bodoquena, Estado de Mato Grosso do Sul, Brasil
}

\author{
Presence of Loxosceles similis Moenkhaus, 1898 (Araneae, Sicariidae) in \\ Bodoquena Range, State of Mato Grosso do Sul, Brazil
}

\section{Rute Maria Gonçalves de Andrade' ${ }^{1}$ Eunice Aparecida Bianchi Galati ${ }^{2}$ e Denise Vilarinho Tambourgi ${ }^{1}$}

Resumo O veneno das aranhas do gênero Loxosceles causa lesão dermonecrótica e induz hemólise intravascular dependente de complemento, configurando um quadro clínico de intensa gravidade. No Brasil, as espécies $L$. gaucho $L$. intermedia e $L$. laeta, presentes no ambiente antrópico, têm sido apontadas como principais agentes do loxoscelismo. Além destas, existem outras espécies, que por predominarem no ambiente natural, não têm sido avaliadas quanto ao risco à saúde do homem, como é o caso de Loxosceles similis. O desenvolvimento de projeto de pesquisa, na Serra da Bodoquena, para observações ecológicas e identificação de insetos de interesse médico, possibilitou a captura de espécimens de Loxosceles similis na Serra da Bodoquena, Município de Bonito, Estado do Mato Grosso do Sul, Brasil, nas grutas Pitangueiras e do Lago Azul. Os parâmetros para identificação, características ambientais do habitat da espécie, bem como atualização de sua distribuição geográfica são objetos deste trabalho.

Palavras-chaves: Loxosceles similis. Loxoscelismo. Cavernas. Mato Grosso do Sul. Serra da Bodoquena.

Abstract The venom of Loxosceles spiders causes dermonecrotic lesion and induces complement-dependent intravascular haemolysis that characterizes a severe systemic effect. In Brazil, L. gaucho, L. intermedia and $L$. laeta, present in the anthropic environment, have been pointed out as the most important agents of the loxoscelism. Besides these species there are others that, by predominating in the natural environment, have not been evaluated regarding human health risk, as in the case of Loxosceles similis. The development of a research project in Bodoquena Range, for ecological observation and identification of insects of medical interest, enabled the capture of Loxosceles similis specimens in the "Pitangueiras" cave and "Lago Azul" cave, in Bodoquena Range, municipality of Bonito, State of Mato Grosso do Sul, Brazil. The objectives of this study were to define the parameters for identification, environmental features of the habitat of this species, as well as an update of its geographical distribution.

Key-words: Loxosceles similis. Loxoscelism. Cave. State of Mato Grosso do Sul. Bodoquena Range.

As Aranhas do gênero Loxosceles são agentes da mais grave forma de araneísmo no Brasil, o Loxocelismo, que representou $36,6 \%$ dos casos de acidentes com aranhas diagnosticados e notificados ao Ministério da Saúde em $1998^{3}$, notadamente nas regiões Sudeste e Sul do país. O veneno destas aranhas causa lesão dermonecrótica e induz hemólise intravascular dependente de complemento, configurando um quadro clínico de intensa gravidade ${ }^{13}$. No Brasil, Loxosceles gaucho, Loxosceles intermedia e Loxosceles laeta, presentes no ambiente antrópico, são consideradas os principais agentes etiológicos do loxoscelismo. Além destas, existem outras espécies que por predominarem no ambiente natural, não têm sido avaliadas quanto ao risco à saúde do homem, como é o caso de Loxosceles similis.

Em ambientes naturais, espécies de Loxosceles têm sido assinaladas em grutas. Ecótopos estes, que atraem um contigente considerável de visitantes como o da Serra da Bodoquena, importante polo turístico do Estado do Mato Grosso do Sul, Brasil 6 , especialmente, devido à presença da área cárstica da região de Bonito. De acordo com Karmann e Sanchéz, $1979^{8}$, a Província Espeleológica da Serra da Bodoquena, com área de $4.660 \mathrm{~km}^{2}$, situa-se no Sudoeste do Mato Grosso do Sul, na unidade geomorfológica Planalto da Bodoquena.

\footnotetext{
1. Laboratório de Imunoquímica do Instituto Butantan, São Paulo, Brasil. 2. Departamento de Epidemiologia da Faculdade de Saúde Pública da Universidade de São Paulo, São Paulo, SP

Suporte Financeiro FAPESP processo № 97/0638-3 e processo № 1998-03155-3

Endereço para correspondência: Dra. Rute Maria Gonçalves de Andrade. Laboratório de Imunoquímica/Instituto Butantan. Av. Vital Brazil 1500, 05508-900,

São Paulo, SP, Brasil.

Tel: 5511 3726-7222 r: 2231/2027, Fax: $55113726-7222$ r: 2162.

e-mail: rutemga@netscape.net

Recebido para publicação em 29/6/2000.
} 
Caracteriza-se pela presença de cavernas com grandes salões que podem, em alguns casos, levar ao lençol freático, formando lagos, como a gruta do Lago Azul.

As entradas das cavernas de Bonito, em geral, são bastante amplas, permitindo entrada de luz em grande extensão e conseqüentemente, redução da umidade relativa do ar, que pode chegar a valores mais baixos (78\% a $96 \%$ ) do que os registrados nas cavernas em geral $^{714}$. Assim sendo, este ambiente é favorável à presença de troglófilos de regiões de entrada e de penumbra, o que justifica portanto, o encontro de Loxosceles.

O desenvolvimento de projeto de pesquisa na Serra da Bodoquena, para identificação e observações ecológicas sobre insetos de interesse médico, possibilitou a captura de espécimes de Loxoesceles similis em duas grutas do Município de Bonito, cujos parâmetros para identificação, características ambientais do habitat da espécie, bem como atualização de sua distribuição geográfica são objetos deste trabalho.

\section{MATERIAL E MÉTODOS}

Área de Captura. As capturas foram realizadas na

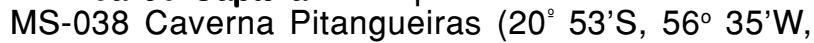
Bonito) $)^{12}$ e na MS-2 Caverna Lago Azul (21 $1^{\circ} 08^{\prime} S, 56^{\circ} 35^{\prime} \mathrm{W}$, Bonito $)^{12}$, município de Bonito, Mato Grosso do Sul.

Método de coleta. As aranhas foram capturadas, manualmente, em janeiro de 1999, acondicionadas vivas em recipientes plásticos e levadas ao Biotério de Criação e Manutenção de Aranhas do Laboratório de Imunoquímica do Instituto Butantan, onde têm sido mantidas.

Os exemplares mortos, devidamente identificados, foram conservados em álcool $70 \%$ e encontram-se no referido laboratório. A espécie foi determinada pelo exame de 2 machos, uma vez que os demais exemplares permanecem vivos, fazendo parte da criação.

A morfometria foi realizada em microscópio esteroscópico LEICA $®$ MZ6 em ocular 10x dotada de retículo com escala milimétrica. Os caracteres analisados foram: largura e comprimento do cefalotórax, comprimento total do palpo e das pernas I, II, III, IV, bem como, comprimento dos artículos do palpo (fêmur, patela, tíbia, tarso) e dos artículos das pernas (fêmur, patela, tíbia, metatarso e tarso).

\section{RESULTADOS}

Embora a presença de Loxosceles similis na MS38 Caverna Pitangueiras, tenha sido considerada comum (presença de dezenas a centenas de exemplares), de acordo com a designação de Trajano e Gnaspini, $1990^{15}$, capturou-se apenas 8 espécimes de Loxosceles similis ( 3 machos jovens, 5 fêmeas jovens), próximo a entrada, local onde era possível perceber luminosidade de baixa intensidade, vinda da abertura da caverna. A maioria das aranhas observadas estava presente sob pedras e em fendas.

Na MS-2 Caverna Lago Azul capturou-se, também na entrada da mesma, em local com luminosidade de baixa intensidade, apenas uma fêmea, próxima a ooteca, cujos aranhiços estavam dispersos, próximos a ela.

Os exemplares de L. similis, encontrados em ambas as cavernas visitadas, apresentam colorido geral marrom com o cefalotórax e as pernas pouco pigmentados.

A fêmea da MS-2 Caverna Lago Azul esta sendo mantida em criadouro e portanto sua morfometria, bem como a análise de seus receptáculos seminais não foi efetuada para identificação específica; porém, fora identificada também como L. similis, uma vez que, acasalada com macho capturado na MS-38 Caverna Pitangueiras produziu ooteca com ovos viáveis.

Os cefalotórax dos machos 1 e 2, capturados na MS-38 Caverna Pitangueiras, medem 5,23mm e $4,94 \mathrm{~mm}$ de comprimento e $3,75 \mathrm{~mm}$ e $2,96 \mathrm{~mm}$ de largura, respectivamente. As medidas dos artículos (perna I, II, III, IV e palpo) para os dois machos são apresentados na Tabela 1.

Tabela 1- Medidas, em mm, dos artículos das pernas I, II, III, IV e do palpo dos machos 1 e 2 de L. similis, capturados na Caverna Pitangueiras, município de Bonito, Estado do Mato Grosso do Sul.

\begin{tabular}{|c|c|c|c|c|c|c|c|c|c|c|}
\hline & \multicolumn{2}{|c|}{ Perna I } & \multicolumn{2}{|c|}{ Perna II } & \multicolumn{2}{|c|}{ Perna III } & \multicolumn{2}{|c|}{ Perna IV } & \multicolumn{2}{|c|}{ Palpo } \\
\hline & 1 & 2 & 1 & 2 & 1 & 2 & 1 & 2 & 1 & 2 \\
\hline Fêmur & 9,18 & 9,68 & 10,37 & 11,46 & 8,15 & 9,28 & 9,28 & 10,27 & 2,27 & 2,37 \\
\hline Patela & 1,48 & 1,28 & 1,58 & 1,48 & 1,48 & 1,19 & 1,48 & 1,48 & 0,59 & 0,79 \\
\hline Tíbia & 9,68 & 11,65 & 12,25 & 13,73 & 7,80 & 8,40 & 10,86 & 10,07 & 1,09 & 1,18 \\
\hline Metatarso & 10,76 & 10,86 & 13,43 & 13,43 & 9,28 & 9,59 & 10,96 & 11,65 & - & - \\
\hline Tarso & 2,07 & 2,07 & 2,37 & 2,86 & 1,78 & 1,88 & 2,27 & 2,27 & 0,95 & 0,99 \\
\hline Total & 33,17 & 35,54 & 40,0 & 42,96 & 28,49 & 30,34 & 34,85 & 35,74 & 4,90 & 5,33 \\
\hline
\end{tabular}




\section{DISCUSSÃO}

Os exemplares de $L$. similis encontrados em ambas as cavernas, evidenciaram discreta despigmentação do colorido geral do corpo, bem como, um alongamento das pernas, quando comparados com exemplares de outras espécies de Loxosceles que habitam ambientes externos às cavernas. Os dois machos utilizados para identificação específica exibem a fórmula das pernas 2, 4, 1, 3, que está de acordo com a descrição original de Moenkhaus, $1898^{10}$ e segundo Gertsch, $1967^{4}$.

A despeito da importância médica que as espécies de Loxosceles representam e do crescente turismo nas áreas cársticas brasileiras, sua presença nestas regiões, não tem sido objeto de estudo. Relatos da presença destas aranhas restringem-se a citações referentes a estudos que objetivaram conhecer a composição da fauna cavernícola brasileira ${ }^{2561516}$.

A presença de Loxosceles na caverna Pitangueiras já havia sido anteriormente registrada por Gnaspini \& Trajano, 19946, porém, sem identificação da espécie. Além disso, os autores a consideraram rara, quanto à densidade, diferentemente do observado para $L$. similis, no presente trabalho. Na gruta do Lago Azul, a presença desta espécie foi registrada também por Gnaspini \& Trajano, 19946 .

A distribuição geográfica de Loxosceles similis, pode ser portanto atualizada, incluindo-se as localidades cársticas em que já fora registrada:
Loxosceles similis Moenkhaus 1898: Iguape (SP) ${ }^{9}$; Altinópolis (SP) ${ }^{1}$; Ubá (MG) ${ }^{4}$; Viçosa (MG) ${ }^{4}$; Ouro Preto $(\mathrm{MG})^{4}$; Minas de Serrinha (MG) ${ }^{4}$; Diamantina (MG) caverna MG- 274 Lapa do Morro Redondo (19 32'04"S-44 02'54'W, Matozinhos, MG) ${ }^{11}$; caverna MG-426 Lapa Vermelha I (19³6'42"S-43⒌'44'W, Pedro Leopoldo, MG) ${ }^{11}$; caverna MG-428 Lapa

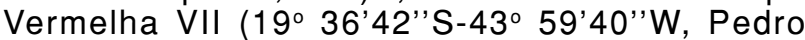
Leopoldo, MG) 11 ; Aurá (PA) ${ }^{9}$ caverna GO-29 Jaguatirica $\left(15^{\circ} 28^{\prime} \mathrm{S}-47^{\circ} 03^{\prime} \mathrm{W}\right.$, Formosa, GO) ${ }^{6}$; caverna BA-52 Padre (13० $13^{\prime} \mathrm{S}-44^{\circ} 04^{\prime} \mathrm{W}$, Santana, BA) ${ }^{6}$; caverna MS-2 Lago Azul ( $21^{\circ} 08^{\prime} S-56^{\circ} 35^{\prime} \mathrm{W}$, Bonito MS $)^{6}$ e MS-038 Caverna Pitangueiras $\left(20^{\circ}\right.$ $53^{\prime} \mathrm{S}, 56^{\circ} 35^{\prime} \mathrm{W}$, Bonito).

A ampla distribuição de $L$. similis no ambiente cavernícola, confirma as observações de Trajano ${ }^{12}$ que se refere às Loxosceles como as aranhas mais comuns nas regiões cársticas brasileiras, provavelmente por se adaptarem bem ao ambiente hipógeo.

Assim sendo, o incremento turístico nessas regiões associado à importância médica destas aranhas justifica estudos mais detalhados quanto ao aspecto troglófilo das Loxosceles, no sentido de entender sua relação com este ecótopo, e, deste modo, conhecer os possíveis riscos de acidentes para freqüentadores.

\section{AGRADECIMENTOS}

À Bióloga Márcia Bicudo do Departamento de Epidemiologia da Faculdade de Saúde Pública - Universidade de São Paulo, pela contribuição na realização da morfometria dos espécimes.

\section{REFERÊNCIAS BIBLIOGRÁFICAS}

1. Camargo HFA. Sobre algumas aranhas que ocorrem no Brasil, com descrição de um alótipo (Arachnida-Araneae). Papéis Avulsos do Departamento de Zoologia 11: 301-340, 1953.

2. Dessen BEM, Eston VR, Silva MS, Temperini-Beck MT, Trajano E. Levantamento preliminar da fauna de cavernas de algumas regiões do Brasil. Ciência e Cultura 32: 714-725, 1980.

3. Fundação Nacional de Saúde. Manual de Diagnóstico e Tratamento de Acidentes por Animais Peçonhentos. Ministério da Saúde, Brasília 49-61, 1998.

4. Gertsch WJ. The spider genus Loxosceles in South America (Araneae, Scytodidae). Bulletin of American Museum of Natural History 136: 117-178, 1967.

5. Gnaspini P, Trajano E. Brazilian cave invertebrates with a checklist of troglomorphic taxa. Revista Brasileira de Entomologia 38: 549584, 1994

6. Gnaspini P, Trajano E, Sánchez LH. Província espeleológica da Serra da Bodoquena, MS: exploração, topografia e biologia. Espeleo-Tema 17: 19-44, 1994.

7. Godoy NM. Nota sobre a fauna cavernícola de Bonito, MS. Espeleo-Tema 16: 41-74, 1986.

8. Karmann I, Sánchez LE. Distribuição das rochas carbonáticas e províncias espeleológicas do Brasil. Espeleo-Tema 13: 105-167, 1979.
9. Mello-Leitão CF. Algumas aranhas da região Amazônica. Boletim do Museu Nacional do Rio de Janeiro 25: 1-12, 1944.

10. Moenkhaus WJ. Contribuição para o conhecimento das aranhas de São Paulo. Revista do Museu Paulista 3: 77-112, 1898.

11. Pinto-da-Rocha R. Sinopse da Fauna cavernícola do Brasil (19071994). Papéis avulsos de Zoologia, 39: 61-173, 1995.

12. Sociedade Brasileira de Espeleologia. Cadastro Nacional de Cavidades Naturais. Índice de dados sobre as cavernas do Brasil. São Paulo, 1989.

13. Tambourgi DV, Morgan BP, Gonçalves-de-Andrade RM, Magnoli FC, van den Berg CW. Loxosceles intermedia spider envenomation induces activation of an endogenous metalloproteinase, resulting in cleavage of glycophorins from the erythrocyte surface and facilitating complement-mediated lysis. Blood 95: 683-691, 2000.

14. Trajano E. Fauna cavernícola brasileira: Composição e caracterização preliminar. Revista Brasileira de Zoologia 3: 533-561, 1987.

15. Trajano E, Gnaspini-Neto P. Composição da fauna cavernícola brasileira, com uma análise preliminar dos táxons. Revista Brasileira de Zoologia 7: 383-407, 1990.

16. Trajano E, Moreira JRA. Estudo da fauna de cavernas da Província Espeleológica Arenítica Altamira-Itaituba. Revista Brasileira de Biologia 51: 13-29, 1991. 